\title{
Group Action on Fuzzy Modules
}

\author{
Mohammad Yamin', Poonam Kumar Sharma² \\ ${ }^{1}$ Faculty of Economics and Administration, King Abdulaziz University, Jeddah, Saudi Arabia \\ ${ }^{2}$ Department of Mathematics, D.A.V. College, Jalandhar City, India \\ Email: myamin@kau.edu.sa, pksharma@davjalandhar.com
}

Received 27 December 2015; accepted 15 March 2016; published 18 March 2016

Copyright (C) 2016 by authors and Scientific Research Publishing Inc.

This work is licensed under the Creative Commons Attribution International License (CC BY). http://creativecommons.org/licenses/by/4.0/

(c) (i) Open Access

\begin{abstract}
In this article, we introduce the notion of fuzzy G-module by defining the group action of $G$ on a fuzzy set of a Z-module $M$. We establish the cases in which fuzzy submodules also become fuzzy Gsubmodules. Notions of a fuzzy prime submodule, fuzzy prime G-submodule, fuzzy semi prime submodule, fuzzy G-semi prime submodule, G-invariant fuzzy submodule and G-invariant fuzzy prime submodule of $M$ are introduced and their properties are described. The homomorphic image and pre-image of fuzzy G-submodules, G-invariant fuzzy submodules and G-invariant fuzzy prime submodules of $M$ are also established.
\end{abstract}

\section{Keywords}

Fuzzy Submodule (FSM), Fuzzy Prime Submodule (FPSM), Fuzzy Semi Prime Submodule, Group Action (FSPSM), G-Invariant Submodule, G-Module Homomorphism

\section{Introduction and Main Results}

The concept of fuzzy subset of a non-empty set was introduced by Zadeh [1] who introduced the notion of a fuzzy set as a method of representing uncertainty in real physical world. Following this landmark discovery, a number of studies of Fuzzy Modules and their applications have emerged. In particular, Negoita and Ralescu in [2] introduced and examined the notion of a fuzzy submodule of a module. Since then, different types of fuzzy submodules have been investigated in the last three decades. Juncheol Han in [3] has studied group actions in regular rings. The notion of group action on fuzzy subset of a ring was defined and studied by Sharma in [4] [5].

Let $\mathrm{X}$ be a non-empty set. A mapping $\mu: \mathrm{X} \rightarrow[0,1]$ is called a fuzzy subset of $\mathrm{X}$. Rosenfeld [6] applied the concept of fuzzy sets to the theory of groups and defined the concept of fuzzy subgroups of a group. Since then, many papers concerning various fuzzy algebraic structures have appeared in the literature. As a generalization of a fuzzy set, the concept of an intuitionistic fuzzy set was introduced by Atanassov [7]. Further results on these and other aspects of fuzzy modules can be found in [8]-[17]. 
In this paper, we define the group action on fuzzy subset of a module over the ring of integers and introduce the notion of fuzzy G-modules. Many properties of fuzzy G-modules will be established. The concept of fuzzy G-prime submodules will be introduced and studied. Following the definition of G-invariant submodule of a module $\mathrm{M}$, we define and study G-invariant fuzzy submodule and G-invariant fuzzy prime submodule of a module M. The homomorphic image and pre-image of fuzzy G-modules will be established. A number of associated results will be obtained.

\section{Preliminaries Knowledge and Results}

We recall some definitions and results for the smooth flow of our assertions and results. Throughout the paper, unless otherwise stated, $\mathrm{R}$ will denote a commutative ring with unity and $\mathrm{M}$ an R-module.

Definition (2.1) [18] Let $\mu$ and $v$ be any two fuzzy sets of an R-module, then

(i) $\mu \subseteq v$ if and only if $\mu(x) \leq v(x)$ for all $x \in \mathrm{M}$;

(ii) $\mu=v$ if and only if $\mu(x)=v(x)$ for all $x \in \mathrm{M}$;

(iii) $(\mu \cap v)(x)=\min \{\mu(x), v(x)\}$ for all $x \in \mathrm{M}$;

(iv) $(\mu \cup v)(x)=\max \{\mu(x), v(x)\}$ for all $x \in \mathrm{M}$;

(v) $(\mu \times v)(x, y)=\min \{\mu(x), v(y)\}$ for all $x, y \in \mathrm{M}$.

Definition (2.2) [19] Let $\mathrm{M}$ be an R-module. Then the fuzzy set $\mu$ of $\mathrm{M}$ is called a fuzzy submodule (FSM) of $\mathrm{M}$ if

(i) $\mu(0)=1$;

(ii) $\mu(x+y) \geq \min \{\mu(x), v(y)\}, \forall x, y \in \mathrm{M}$;

(iii) $\mu(r x) \geq \mu(x), \forall x \in \mathrm{M}, r \in \mathrm{R}$.

Definition (2.3) [19] Let $\mu$ and $v$ be two fuzzy submodules of an R-module M, then their sum $\mu+v$ and product $\mu v$ are defined as

(i) $(\mu+v)(x)=\operatorname{Sup}_{x=a+b}\{\min \{\mu(a), v(b)\}\}, \forall x \in \mathrm{M}$;

(ii) $(\mu v)(x)=\operatorname{Sup}_{x=\sum_{i<\infty} a_{i} b_{i}} \min _{i}\left\{\min \left\{\mu\left(a_{i}\right), v\left(b_{i}\right)\right\}\right\}, \forall x \in$ M.

Theorem (2.4) [19] Let $\mu$ and $v$ be two fuzzy submodules of an R-module M. Then the sum $\mu+v$ and the product $\mu v$ of $\mu$ and $v$ are also fuzzy submodules of M.

Theorem (2.5) [20] Let $\mu$ and $v$ be two fuzzy submodules of an R-module M. Then $\mu \cap v$ is also a fuzzy submodule of $\mathrm{M}$.

In particular, if $\left\{\mu_{i}: i \in I\right\}$ be a family of fuzzy submodules of an R-module $\mathrm{M}$, then $\bigcap_{i \in I} \mu_{i}$ is also a fuzzy submodule of $\mathrm{M}$.

Theorem (2.6) [20] Let $\mu$ andvbe two fuzzy submodules of an R-module M. Then $\mu \times v$ is also a fuzzy submodule of $\mathrm{M} \times \mathrm{M}$.

\section{Group Action on Fuzzy Modules}

Most of the results below can be extended to an arbitrary commutative ring. We have not been able to remove the restriction to the ring of integers in Lemma (3.3) (iii) and so some results cannot be extended.

Let $\mathrm{M}$ be a module over the ring of integers $\mathrm{Z}$ and $\mathbf{G}$ a finite group which acts on $\mathbf{M}$ (i.e., $\forall g \in \mathrm{G}, x \in \mathrm{M}, x^{g}$ $\left.=g x g^{-1} \in \mathrm{M}\right)$. The identity element of $\mathrm{G}$ is denoted by $e$.

Definition (3.1) A group action of $\mathrm{G}$ on a fuzzy set $\mu$ of a Z-module $\mathrm{M}$ is denoted by $\mu^{g}$ and is defined by $\mu^{g}(x)=\mu\left(x^{g}\right), g \in \mathrm{G}$.

From the definition of group action $\mathrm{G}$ on a fuzzy set, following results are easy to verify.

Lemma (3.2) Let $\mu$ and $v$ be two fuzzy sets of Z-module $M$ and $\mathrm{G}$ a finite group which acts on $\mathrm{M}$. Then 
(i) If $\mu \subseteq v$ then $\mu^{g} \subseteq v^{g}, \forall g \in \mathrm{G}$;

(ii) $(\mu \cup v)^{g}=\left(\mu^{g} \cup v^{g}\right)$;

(iii) $(\mu \cap v)^{g}=\left(\mu^{g} \cap v^{g}\right)$;

(iv) $(\mu \times v)^{g}=\left(\mu^{g} \times v^{g}\right)$;

(v) $\left(\mu^{g_{1}}\right)^{g_{2}}=\mu^{g_{1} g_{2}} \forall g_{1}, g_{2} \in \mathrm{G}$;

(vi) $\left(\mu^{g}\right)^{g^{-1}}=\mu^{e}=\mu, \forall g \in \mathrm{G}$.

Let us now prove

Lemma (3.3) Let $\mathrm{G}$ be a finite group which acts on Z-module M. Then for every $x, y \in \mathrm{M}, g \in \mathrm{G}$ and $r \in \mathrm{Z}$, we
(i) $(x+y)^{g}=x^{g} ? y^{g}$
(ii) $(x y)^{g}=x^{g} y^{g}$
(iii) $(r x)^{g}=r x^{g}$
(iv) $(x, y)^{g}=\left(x^{g}, y^{g}\right)$

Proof: (i) Since $(x+y)^{g}=g(x+y) g^{-1}=g x g^{-1}+g y g^{-1}=x^{g}+y^{g}$.

(ii) $(x y)^{g}=g(x y) g^{-1}=g(x e y) g^{-1}=g\left(x g^{-1} g y\right) g^{-1}=\left(g x g^{-1}\right)\left(g y g^{-1}\right)=x^{g} y^{g}$.

(iii) $(r x)^{g}=g(r x) g^{-1}=g(x+x+\cdots|r|$ times $) g^{-1}=g x g^{-1}+g x g^{-1}+\cdots|r|$ times $=r\left(g x g^{-1}\right)=r x^{g}$.

(iv) $(x, y)^{g}=g(x, y) g^{-1}=\left(g x g^{-1}, g y g^{-1}\right)=\left(x^{g}, y^{g}\right)$.

Proposition (3.4) Let $\mu$ be a fuzzy submodule of Z-module M and G a finite group which acts on $\mathrm{M}$, then $\mu^{g}$ is also a fuzzy submodule of $\mathrm{M}$.

Proof: Clearly, $\mu^{g}(0)=\mu\left(0^{g}\right)=\mu(0)=1$.

Let $x, y \in \mathrm{M}, g \in \mathrm{G}$ and $r \in \mathrm{Z}$ then, by lemma (3.3) (i),

$$
\begin{aligned}
\mu^{g}(x+y) & =\mu\left\{(x+y)^{g}\right\}=\mu\left(x^{g}+y^{g}\right) \\
& \geq \min \left\{\mu\left(x^{g}\right), \mu\left(y^{g}\right)\right\}=\min \left\{\mu^{g}(x), \mu^{g}(y)\right\} .
\end{aligned}
$$

Moreover, $\mu^{g}(r x)=\mu\left\{(r x)^{g}\right\}=\mu\left(r x^{g}\right) \geq \mu\left(x^{g}\right)=\mu^{g}(x)$ by lemma (3.3) (iii).

Hence $\mu^{g}$ is a fuzzy submodule of $\mathrm{M}$.

Remark (3.5) The converse of Proposition (3.4) does not hold.

Example (3.6) Let $\mathrm{M}=\left(\mathrm{Z}_{4}=\{0,1,2,3\},{ }_{4}, \mathrm{x}_{4}\right)$ regarded as $\mathrm{Z}$-module, and a finite group $\mathrm{G}=(\{1,2,3,4\}$, $\times_{5}$ ). Consider a fuzzy set $\mu$ of M given by $\mu(0)=1, \mu(1)=0.4, \mu(2)=0.6, \mu(3)=0.5$. Clearly $\mu$ is not fuzzy submodule of M because $\mu\left(2+{ }_{4} 3\right)=\mu(1)=0.4<0.5=\min \{0.6,0.5\}=\min \{\mu(2), \mu(3)\}$.

Take $g=2$ so that $g^{-1}=3$, then $x^{g}=g x g^{-1}=2 \times_{4} x \times_{4} 3=6 x(\bmod 4)=2 x(\bmod 4)$, we get $\mu^{g}(x)=\mu\left(x^{g}\right)=\left\{\begin{array}{cl}1 & \text { if } x=0,2 \\ 0.6 & \text { if } x=1,3\end{array}\right.$

Now, it is easy to check that $\mu^{g}$ is a fuzzy submodule of M.

Definition (3.7) Let $\mu$ be a fuzzy set of Z-module M and G be a finite group which acts on $\mathrm{M}$. Then $\mu$ is called a fuzzy G-submodule of $\mathrm{M}$ if $\mu^{g}$ is fuzzy submodule of $\mathrm{M}$ for all $g \in \mathrm{G}$.

Remark (3.8) (i) From definition (3.7) we see that every fuzzy G-submodule is also a fuzzy submodule, for $\mu^{e}=\mu$.

(ii) Note that the fuzzy set $\mu$ in example (3.6) is not a fuzzy G-submodule of $\mathrm{M}$, for $\mu^{e}=\mu$ is not a fuzzy submodule of $\mathrm{M}$.

Theorem (3.9) Let $\mu$ be a fuzzy submodule of Z-module M and $\mathrm{G}$ be a finite group which acts on $\mathrm{M}$, then $\mu$ is a fuzzy G-submodule of $\mathrm{M}$ if and only if for every $g \in \mathrm{G}, \mu^{g}$ satisfies the following conditions:

(i) $\mu^{g}(0)=1$; (ii) $\mu^{g}(r x+s y) \geq \min \left\{\mu^{g}(x), \mu^{g}(y)\right\} \quad \forall r, s \in \mathrm{Z} ; x, y \in \mathrm{M}$. 
Proof: Firstly, let $\mu$ be a FSM of Z-module M and $g \in \mathrm{G}$ such that $\mu^{g}$ satisfies the given conditions.

Substituting $r=s=1$ in condition (ii), we get

$$
\mu^{g}(1 \cdot x+1 \cdot y)=\mu^{g}(x+y) \geq \min \left\{\mu^{g}(x), \mu^{g}(y)\right\} .
$$

Also, putting $s=0$ in condition (ii) we get

$$
\begin{aligned}
\mu^{g}(r x)=\mu^{g}(r x+0 \cdot y) & =\mu^{g}(r x+0 \times 0) \geq \min \left\{\mu^{g}(x), \mu^{g}(0)\right\} \\
& =\min \left\{\mu^{g}(x), 1\right\}=\mu^{g}(x) .
\end{aligned}
$$

Thus $\mu^{g}(r x) \geq \mu^{g}(x)$.

Therefore, $\mu^{g}$ is a fuzzy submodule of M and hence $\mu$ is a fuzzy G-submodule of M.

Conversely, let $\mu$ be a fuzzy G-submodule of $\mathrm{M}$, and $g \in \mathrm{G}$. To establish (i) and (ii), we only need to prove (ii).

Let $r, s \in \mathrm{Z}$ and $x, y \in \mathrm{M}$. Then

$$
\begin{aligned}
\mu^{g}(r x+s y) & =\mu\left\{(r x+s y)^{g}\right\}=\mu\left\{(r x)^{g}+(s y)^{g}\right\}=\mu\left\{r x^{g}+s y^{g}\right\} \\
& \geq \min \left\{\mu\left(r x^{g}\right), \mu\left(s y^{g}\right)\right\} \geq \min \left\{\mu\left(x^{g}\right), \mu\left(y^{g}\right)\right\} \\
& =\min \left\{\mu^{g}(x), \mu^{g}(y)\right\} .
\end{aligned}
$$

Thus $\mu^{g}(r x+s y) \geq \min \left\{\mu^{g}(x), \mu^{g}(y)\right\}$. Hence the result proved.

Proposition (3.10) Let $\mu$ and $v$ be two fuzzy G-submodules of a Z-module M and G be a finite group which acts on $\mathrm{M}$. Then $\mu \cap v$ is also a fuzzy G-submodule of $\mathrm{M}$.

Proof: Since $\mu$ and $v$ are fuzzy G-submodules of M. Therefore $\mu^{g}$ and $v^{g}$ are fuzzy submodules of M for all $g \in \mathrm{G}$ which implies that $\mu^{g} \cap v^{g}$ is fuzzy submodule of M [By Theorem (2.5)].

Thus $(\mu \cap v)^{g}$ is fuzzy submodule of M for all $g \in \mathrm{G}$ [By Lemma (3.2) (ii)]. Hence $\mu \cap v$ is also a fuzzy G-submodule of $\mathrm{M}$.

Proposition (3.11) Let $\mu$ and $v$ be two fuzzy G-submodules of Z-module M and G be a finite group which acts on $\mathrm{M}$. Then $\mu \times v$ is also a fuzzy $\mathrm{G}$-submodule of $\mathrm{M} \times \mathrm{M}$.

Proof: Since $\mu$ and $v$ are fuzzy G-submodules of M. Therefore $\mu^{g}$ and $v^{g}$ are fuzzy submodules of M for all $g \in \mathrm{G}$ which implies that $\mu^{g} \times v^{g}$ is fuzzy submodule of $\mathrm{M} \times \mathrm{M}$ [By Theorem (2.6)]. Thus $(\mu \times v)^{g}$ is a fuzzy submodule of $\mathrm{M} \times \mathrm{M}$ for all $g \in \mathrm{G}$ [By Lemma (3.2) (iv)]. Hence $\mu \times v$ is also a fuzzy G-submodule of $\mathrm{M}$ $\times$ M.

We now define fuzzy prime submodule (FPSM) and fuzzy G-prime submodule of M.

Definition (3.12) A fuzzy submodule $\gamma$ of Z-module $M$ is called a fuzzy prime submodule if for any two fuzzy submodules $\mu, v$ of M such that $\mu v \subseteq \gamma$ implies that either $\mu \subseteq \gamma$ or $v \subseteq \gamma$.

Lemma (3.13) Let $\mu$ and $v$ be two fuzzy prime submodules of Z-module M. Then $\mu \cap v$ is fuzzy prime submodule of $\mathrm{M}$ if and only if either $\mu \subseteq v$ or $v \subseteq \mu$.

Proof. We know that $\mu \nu \subseteq \mu \cap v$. Therefore, $\mu \cap v$ is fuzzy prime submodule of $\mathrm{M}$ if and only if either $\mu \subseteq \mu$ $\cap v$ or $v \subseteq \mu \cap v$. But $\mu \cap v \subseteq \mu$ and $\mu \cap v \subseteq \mu$. Thus $\mu \cap v$ is fuzzy prime submodule of $\mathrm{M}$ if and only if either $\mu=\mu \cap v$ or $v=\mu \cap v$, i.e., either $\mu \subseteq v$ or $v \subseteq \mu$.

Remark (3.14) From Lemma (3.13) we infer that in general intersection of two fuzzy prime submodules need not to be a fuzzy prime submodule.

Theorem (3.15) Let $\gamma$ be a fuzzy prime submodule of a Z-module M and G be a finite group which acts on M. Then $\gamma^{g}$ is also a fuzzy prime submodule of $\mathrm{M}$.

Proof: Let $\mu$ and $v$ be fuzzy submodules of M such that $\mu \nu \subseteq \gamma^{g}$. Now, we claim that $\mu^{g^{-1}} v^{g^{-1}} \subseteq \gamma$. It is sufficient to show that $\mu^{g^{-1}} v^{g^{-1}}(x) \leq \gamma(x), \forall x \in \mathrm{M}$.

Now 


$$
\begin{aligned}
\mu^{g^{-1}} v^{g^{-1}}(x) & =\operatorname{Sup}_{x=\sum_{i<b_{i}}}\left\{\min _{i}\left\{\min \left(\mu^{g^{-1}}\left(a_{i}\right), v^{g^{-1}}\left(b_{i}\right)\right)\right\}\right\} \\
& \left.=\operatorname{Sup}_{x=\sum_{i<\alpha} a_{i}}\left\{\min _{i}\left\{\min \left(\mu\left(a_{i}^{g^{-1}}\right), v\left(b_{i}^{g^{-1}}\right)\right)\right)\right\}\right\} \\
& =\operatorname{Sup}_{x^{g^{-1}}=\sum_{i<\alpha} a_{i}^{g^{-1}} b_{i}^{g^{-}}}\left\{\min _{i}\left\{\min \left(\mu\left(a_{i}^{g^{-1}}\right), v\left(b_{i}^{g^{-1}}\right)\right)\right\}\right\} \\
& =(\mu \nu)\left(x^{g^{-1}}\right) \leq \gamma^{g}\left(x^{g^{-1}}\right)=\gamma(x) .
\end{aligned}
$$

Thus $\mu^{g^{-1}} v^{g^{-1}}(x) \leq \gamma(x)$ which implies $\mu^{g^{-1}} v^{g^{-1}} \subseteq \gamma$. But $\gamma$ is a fuzzy prime submodule of M. Therefore, either $\mu^{g^{-1}} \subseteq \gamma$ or $v^{g^{-1}} \subseteq \gamma$ which amounts to $\mu \subseteq \gamma^{g}$ or $v \subseteq \gamma^{g}$. Hence $\gamma^{g}$ is a fuzzy prime submodule of $\mathrm{M}$.

Definition (3.16) Let $\gamma$ be a fuzzy prime submodule of Z-module M and G be a finite group which acts on M. Then $\gamma$ is called a fuzzy G-prime submodule of M if $\gamma^{g}$ is fuzzy prime submodule of M for all $g \in \mathrm{G}$.

Remark (3.17) If we denote $\gamma^{\mathrm{G}}=\bigcap_{g \in \mathrm{G}} \gamma^{g}$, where $\gamma$ is a fuzzy prime submodule of M. Then $\gamma^{\mathrm{G}}$ need not be a fuzzy prime submodule of $\mathrm{M}$, because intersection of fuzzy prime submodules of $\mathrm{M}$, in general, is not a fuzzy prime submodule of M (See Remark (3.14)).

Definition (3.18) A fuzzy submodule $\gamma$ of Z-module M is called a fuzzy semi prime submodule (FSPSM) if for all fuzzy submodules $\mu$ of M such that $\mu^{2} \subseteq \gamma$ implies that $\mu \subseteq \gamma$.

Theorem (3.19) Intersection of two fuzzy prime submodules of a Z-module M is always a fuzzy semi prime submodule of $\mathrm{M}$.

Proof. Let $\gamma_{1}, \gamma_{2}$ be two fuzzy prime submodule of a Z-module M. Let $\mu$ be a fuzzy submodule of M such that $\mu^{2} \subseteq \gamma_{1} \cap \gamma_{2}$. Then we have $\mu^{2} \subseteq \gamma_{1}$ and $\mu^{2} \subseteq \gamma_{2}$. But $\gamma_{1}$ and $\gamma_{2}$ are FPSMs of M. Therefore, $\mu \subseteq \gamma_{1}$ and $\mu \subseteq \gamma_{2}$ which implies that $\mu \subseteq \gamma_{1} \cap \gamma_{2}$. Hence $\gamma_{1} \cap \gamma_{2}$ is fuzzy semi prime submodule of M.

Theorem (3.20) Let $\gamma$ be a fuzzy semi prime submodule of a Z-module M and G be a finite group which acts on $\mathrm{M}$. Then $\gamma^{g}$ is also a fuzzy semi prime submodule of $\mathrm{M}$.

Proof. Let $\mu_{2}$ be any fuzzy submodule of $\mathrm{M}$ and $g \in \mathrm{G}$ be any element such that $\mu^{2} \subseteq \gamma^{g}$, then $\left(\mu^{2}\right)^{g^{-1}}=\left(\mu^{g^{-1}}\right)^{2} \subseteq \gamma$ [follows from Theorem (3.15)], but $\gamma$ is fuzzy semi prime submodule. Therefore, $\mu^{g^{-1}} \subseteq \gamma$, implying $\mu \subseteq \gamma^{g}$. Hence $\gamma^{g}$ is fuzzy semi prime submodule of M.

Definition (3.21) Let $\gamma$ be a fuzzy semi prime submodule of Z-module M and $G$ be a finite group which acts on $\mathrm{M}$. Then $\gamma$ is called a fuzzy G-semi prime submodule (FGSPSM) of M if $\gamma^{\beta}$ is fuzzy semi prime submodule of M for all $g \in \mathrm{G}$.

Theorem (3.22) If we denote $\gamma^{\mathrm{G}}=\bigcap_{g \in \mathrm{G}} \gamma^{g}$, where $\gamma$ is a fuzzy semi prime submodule of M. Then $\gamma^{\mathrm{G}}$ is a fuzzy G-semi prime submodule of M.

Proof. Let $\mu$ be a fuzzy submodule of M such that $\mu^{2} \subseteq \gamma^{G}=\bigcap_{g \in \mathrm{G}} \gamma^{g} \Rightarrow \mu^{2} \subseteq \gamma^{g}, \forall g \in \mathrm{G} \Rightarrow \mu \subseteq \gamma^{g}, \forall g \in \mathrm{G}$ $\Rightarrow \mu \subseteq \bigcap_{g \in \mathrm{G}} \gamma^{g}=\gamma^{G}$. Hence $\gamma^{G}$ is a fuzzy G-semi prime submodule of $\mathrm{M}$.

Following the definition of G-invariant submodule of a module M, we define G-invariant fuzzy submodule and G-invariant fuzzy prime submodule of Z-module M.

Definition (3.23) Let $\mu$ be a fuzzy submodule of a Z-module $M$ and $\mathrm{G}$ be a finite group. Then $\mu$ is said to be G-invariant fuzzy submodule of $\mathrm{M}$ if and only if $\mu^{g}(x)=\mu\left(x^{g}\right) \geq \mu(x) \forall x \in \mathrm{M}, g \in \mathrm{G}$.

Proposition (3.24) Let $\mu$ be a fuzzy submodule of a Z-module M and $\mathrm{G}$ be a finite group which acts on $\mathrm{M}$. Then $\mu$ is G-invariant fuzzy submodule of M if and only if $\mu^{g}=\mu, \forall g \in \mathrm{G}$.

Proof: For $x \in \mathrm{M}, g \in \mathrm{G}$, we have $\mu(x)=\mu\left\{\left(x^{g}\right)^{g^{-1}}\right\} \geq \mu\left(x^{g}\right) \geq \mu(x)$ implies that $\forall g \in \mathrm{G}$, we have 
$\mu(x)=\mu\left(x^{g}\right)=\mu^{g}(x)$. Hence $\mu^{g}=\mu$.

Lemma (3.25) Let $\mu$ be a fuzzy submodule of a Z-module M and G be a finite group which acts on M. Let $\mu^{\mathrm{G}}=\bigcap_{g \in \mathrm{G}} \mu^{g}$ where $\mu^{\mathrm{G}}(x)=\min \left\{\mu\left(x^{g}\right): g \in \mathrm{G}\right\}$. Then $\mu^{\mathrm{G}}$ is the largest G-invariant fuzzy submodule of M.

Proof: Since $\mu$ be a fuzzy submodule of Z-module of M and so $\mu^{g}$ is fuzzy submodule of M for all $g \in \mathrm{G}$. Also,intersection of fuzzy submodules of $\mathrm{M}$ is a fuzzy submodule of $\mathrm{M}$. Now,

$$
\begin{aligned}
\mu^{G}\left(x^{g}\right) & =\min \left\{\mu^{h}\left(x^{g}\right): h \in G\right\}=\min \left\{\mu\left(\left(x^{g}\right)^{h}\right): h \in G\right\} \\
& =\min \left\{\mu\left(x^{g h}\right): h \in G\right\}=\min \left\{\mu\left(x^{g^{\prime}}\right): g^{\prime} \in G\right\}=\mu^{G}(x) .
\end{aligned}
$$

Therefore, $\mu^{G}\left(x^{g}\right)=\mu^{G}(x)$. Thus $\mu^{G}$ is G-invariant fuzzy submodule of M. Further, let $v$ be any G-invariant fuzzy submodule of $\mathrm{M}$ such that $v \subseteq \mu$. Then for $g \in \mathrm{G}$, we get $v\left(x^{g}\right)=v(x) \leq \mu(x)$. Now, $v\left(x^{g}\right)=v(x)=v\left(\left(x^{g}\right)^{g-1}\right) \leq \mu\left(x^{g}\right) \Rightarrow v(x) \leq \min \left\{\mu\left(x^{g}\right): g \in \mathrm{G}\right\}=\mu^{\mathrm{G}}(x)$. Thus $v \subseteq \mu^{\mathrm{G}}$. Hence $\mu^{\mathrm{G}}$ is the largest G-invariant FSM of M.

Proposition (3.26) A fuzzy submodule $\mu$ of Z-module is G-invariant fuzzysubmodule of M if and only if $\mu^{\mathrm{G}}$ $=\mu$.

Proof: This follows from Proposition (3.4) and Proposition (3.24).

Proposition (3.27) Let $\mu$ be fuzzy submodule of Z-module M. Then $\mu^{\mathrm{G}}$ is the largest G-invariant fuzzy submodule of $\mathrm{M}$ contained in $\mu$.

Proof: This follows immediately from Proposition (3.4) and Proposition (3.25)

Theorem (3.28) If $\mu$ and $v$ are G-invariant fuzzy submodules of $\mathrm{M}$, then $\mu+v$ is also a G-invariant fuzzy submodule on $\mathrm{M}$.

Proof. Let $x, y \in \mathrm{M}, g \in \mathrm{G}$ be any elements, then

$$
(\mu+v)^{g}(x)=(\mu+v)\left(x^{g}\right)=\operatorname{Sup}_{x^{g}=a+b}\{\min \{\mu(a), v(b)\}\}=\operatorname{Sup}_{x=a^{g^{-1}}+b^{g^{-1}}}\left\{\min \left\{\mu\left(a^{g^{-1}}\right), v\left(b^{g^{-1}}\right)\right\}\right\}=(\mu+v)(x) \text {. }
$$

Hence $\mu+v$ is a G-invariant fuzzy submodule of $\mathrm{M}$.

Theorem (3.29) If $\mu$ and $v$ are G-invariant fuzzy submodules of $\mathrm{M}$, then $\mu \nu$ is also a G-invariant fuzzy submodule on $\mathrm{M}$.

Proof: Let $x, y \in \mathrm{M}, g \in \mathrm{G}$ be any elements, then

$$
\begin{aligned}
(\mu v)^{g}(x) & =(\mu v)\left(x^{g}\right)=\operatorname{Sup}_{x^{g}=\sum_{i<x} a_{i} b_{i}} \min \left\{\min \left\{\mu\left(a_{i}\right), \mu\left(b_{i}\right)\right\}\right\} \\
& =\operatorname{Sup}_{x=\sum_{i<x} a_{i}{ }^{-1} b_{i} g^{-1}} \min _{i}\left\{\min \left\{\mu\left(a_{i}^{g^{-1}}\right), \mu\left(b_{i}^{g^{-1}}\right)\right\}\right\} \quad \text { [using Lemma (3.2)(ii)] } \\
& =(\mu v)(x) .
\end{aligned}
$$

Hence $\mu v$ is a G-invariant fuzzy submodule of $\mathrm{M}$.

Definition (3.30) A non-constant fuzzy prime submodule $\gamma$ of Z-module M is called an G-invariant fuzzy G-prime submodule of $\mathrm{M}$ if for any two G-invariant fuzzy submodules $\mu$ and $\nu$ of $\mathrm{M}$ such that $\mu \nu \subseteq \gamma$ implies that either $\mu \subseteq \gamma$ or $v \subseteq \gamma$.

Theorem (3.31) If $\gamma$ is a fuzzy prime submodule of a Z-module M, then $\gamma^{G}$ is G-invariant fuzzy G-prime submodule of $\mathrm{M}$.

Proof: Let $\gamma$ be FPSM of M and let $\mu$ and we two G-invariant FSM of M such that $\mu \nu \subseteq \gamma^{G}$. Then $\mu \nu \subseteq \gamma$ because $\gamma^{\mathrm{G}} \subseteq \gamma$. Since $\gamma$ is a fuzzy prime submodule of M, either $\mu \subseteq \gamma$ or $v \subseteq \gamma$, thus either $\mu \subseteq \gamma^{\mathrm{G}}$ or $v \subseteq \gamma^{\mathrm{G}}$. Since $\gamma^{G}$ is the largest G-invariant fuzzy submodule of M contained in $\gamma$. Hence $\gamma^{G}$ is G-invariant fuzzy G-prime submodule of $\mathrm{M}$.

\section{Homomorphism of Fuzzy G-Submodules}

In this section, we study the image and pre image of fuzzy G-submodules under the module homomorphism. 
Lemma (4.1) Let $\mathrm{M}$ and $\mathrm{M}^{\prime}$ be Z-modules and $\mathrm{G}$ be a finite group which acts on $\mathrm{M}$ and $\mathrm{M}^{\prime}$. Let $f: \mathrm{M} \rightarrow \mathrm{M}^{\prime}$ be a mapping defined by $f\left(x^{g}\right)=(f(x))^{g}, \forall x \in \mathrm{M}, g \in \mathrm{G}$. Then $f$ is a module homomorphism. We call the map $f$ as G-module homomorphism.

Proof: Let $x, y \in \mathrm{M}, g \in \mathrm{G}$ and $r \in \mathrm{Z}$ be any elements, then we have

$$
f\left(x^{g}+y^{g}\right)=f\left((x+y)^{g}\right)=(f(x+y))^{g}=(f(x)+f(y))^{g}=(f(x))^{g}+(f(y))^{g}=f\left(x^{g}\right)+f\left(y^{g}\right)
$$

Also $f\left(r x^{g}\right)=f\left((r x)^{g}\right)=(r f(x))^{g}=r(f(x))^{g}=r f\left(x^{g}\right)$.

Hence $f$ is a module homomorphism.

Lemma (4.2) Let $\mathrm{M}$ and $\mathrm{M}^{\prime}$ be $\mathrm{Z}$-modules and $\mathrm{G}$ be a finite group which acts on $\mathrm{M}$ and $\mathrm{M}^{\prime}$. Let $f: \mathrm{M} \rightarrow \mathrm{M}^{\prime}$ be a G-module homomorphism and $\mu$ and $v$ are the fuzzy subsets of $\mathrm{M}$ and $\mathrm{M}^{\prime}$ respectively. Then

(i) $f^{-1}\left(v^{g}\right)=\left(f^{-1}(v)\right)^{g}, \forall g \in \mathrm{G}$;

(ii) $f\left(\mu^{g}\right)=(f(\mu))^{g}, \forall g \in \mathrm{G}$.

Proof. (i) Let $x \in \mathrm{M}$ and $g \in \mathrm{G}$ be any element. Then

$$
f^{-1}\left(v^{g}\right)(x)=v^{g}(f(x))=v\left((f(x))^{g}\right)=v\left(f\left(x^{g}\right)\right)=\left(f^{-1}(v)\right)\left(x^{g}\right)=\left(f^{-1}(v)\right)^{g}(x) .
$$

Hence $f^{-1}\left(v^{g}\right)=\left(f^{-1}(v)\right)^{g}$.

(ii) Let $y \in \mathrm{M}^{\prime}$ and $g \in \mathrm{G}$ be any element. Then

$$
\begin{aligned}
\left(f\left(\mu^{g}\right)\right)(y) & =\operatorname{Sup}\left\{\mu^{g}(x): f(x)=y\right\}=\operatorname{Sup}\left\{\mu\left(x^{g}\right): f(x)=y\right\} \\
& =\operatorname{Sup}\left\{\mu\left(x^{g}\right): f\left(x^{g}\right)=y^{g}\right\} \quad\left[\because f(x)=y \Rightarrow f\left(x^{g}\right)=y^{g}\right] \\
& =(f(\mu))\left(f\left(x^{g}\right)\right)=(f(\mu))\left((f(x))^{g}\right)=(f(\mu))^{g}(f(x))=(f(\mu))^{g}(y) .
\end{aligned}
$$

Hence $\left(f\left(\mu^{g}\right)\right)=(f(\mu))^{g}$.

Theorem (4.3) Let $\mathrm{M}$ and $\mathrm{M}^{\prime}$ be Z-modules on which $\mathrm{G}$ acts and let $f$ be a G-module homomorphism from $\mathrm{M}$ into $\mathrm{M}^{\prime}$. If $v$ be a fuzzy G-submodule of $\mathrm{M}^{\prime}$, then $f^{-1}(v)$ is a fuzzy G-submodule of $\mathrm{M}$.

Proof: Let $v$ be a fuzzy G-submodule of $\mathrm{M}^{\prime}$. To show that $f^{-1}(v)$ is a fuzzy G-submodule of M. It is equivalent to showing that $\left(f^{-1}(v)\right)^{g}$ is a fuzzy submodule of M for all $g \in \mathrm{G}$. But, in view of Lemma (4.2) (i), it is sufficient to show that $f^{-1}\left(\nu^{g}\right)$ is a fuzzy submodule of $\mathrm{M}$ for all $g \in \mathrm{G}$. For $x, y \in \mathrm{M}, r \in \mathrm{Z}$ and $g \in \mathrm{G}$, we have

$$
\begin{aligned}
& f^{-1}\left(v^{g}\right)(0)=v^{g}(f(0))=v^{g}\left(0^{\prime}\right)=v^{g}\left(\left(0^{\prime}\right)^{g}\right)=v^{g}\left(0^{\prime}\right)=1 . \\
& f^{-1}\left(v^{g}\right)(x+y)=v^{g}(f(x+y))=v^{g}(f(x)+f(y))[\because f \text { is a module homomorphism }] \\
& \geq \min \left\{v^{g}(f(x)), v^{g}(f(y))\right\}\left[\because v^{g} \text { is a FSM of } \mathrm{M}^{\prime}\right] \\
& \geq \min \left\{f^{-1}\left(v^{g}\right)(x), f^{-1}\left(v^{g}\right)(y)\right\} .
\end{aligned}
$$

Thus, $f^{-1}\left(v^{g}\right)(x+y) \geq \min \left\{f^{-1}\left(v^{g}\right)(x), f^{-1}\left(v^{g}\right)(y)\right\}$.

Also, $f^{-1}\left(v^{g}\right)(r x)=v^{g}(f(r x))=v^{g}(r f(x))[\because f$ is a module homomorphism $]$

$$
\begin{aligned}
& \quad \geq v^{g}(f(x))\left[\because v^{g} \text { is a FSM of } \mathrm{M}^{\prime}\right] \geq f^{-1}\left(v^{g}\right)(x) \\
& \therefore f^{-1}\left(v^{g}\right)(r x) \geq\left(f^{-1}\left(v^{g}\right)\right)(x) .
\end{aligned}
$$


Therefore, $f^{-1}\left(v^{g}\right)$ and so $\left(f^{-1}(v)\right)^{g}$ is a fuzzy submodule of $\mathrm{M}$.

Hence $f^{-1}(v)$ is a fuzzy G-submodule of $\mathrm{M}$.

Theorem (4.4) Let $\mathrm{M}$ and $\mathrm{M}^{\prime}$ be Z-modules on which $\mathrm{G}$ acts and let $f$ be a bijective G-module homomorphism from $\mathrm{M}$ into $\mathrm{M}^{\prime}$. If $\mu$ is a fuzzy G-submodule of $\mathrm{M}$, then $f(\mu)$ is a fuzzy G-submodule of $\mathrm{M}^{\prime}$.

Proof: Let $\mu$ be a fuzzy G-submodule of $\mathrm{M}$. To show that $f(\mu)$ is a fuzzy G-submodule of $\mathrm{M}^{\prime}$ is equivalent to showing that $(f(\mu))^{g}$ is a fuzzy submodule of $\mathrm{M}^{\prime}$ for all $g \in \mathrm{G}$.

For, in view of Lemma (4.2) (ii), it is sufficient to show that $f\left(\mu^{g}\right)$ is a fuzzy submodule of $\mathrm{M}^{\prime}$ for all $g \in \mathrm{G}$.

$$
\text { Now, } f\left(\mu^{g}\right)\left(0^{\prime}\right)=\operatorname{Sup}\left\{\mu^{g}(z): z \in f^{-1}\left(0^{\prime}\right)\right\}=\mu^{g}((0)=1 .
$$

Then for any $x^{\prime}, y^{\prime} \in \mathrm{M}^{\prime}$ there exists unique $x, y \in \mathrm{M}$ such that $f(x)=x^{\prime}$ and $f(y)=y^{\prime}$.

$$
\begin{aligned}
f\left(\mu^{g}\right)\left(x^{\prime}+y^{\prime}\right) & =f\left(\mu^{g}\right)(f(x)+f(y))=f\left(\mu^{g}\right)(f(x+y)) \\
& =\mu^{g}(x+y)[\because f \text { is bijective G-module homomorphism }] \\
& \geq \min \left\{\mu^{g}(x), \mu^{g}(y)\right\} \\
& =\min \left\{f\left(\mu^{g}\right)(f(x)), f\left(\mu^{g}\right)(f(y))\right\} \\
& =\min \left\{f\left(\mu^{g}\right)\left(x^{\prime}\right), f\left(\mu^{g}\right)\left(y^{\prime}\right)\right\}\left[\because \mu^{g} \text { is a FSM of } \mathrm{M}\right]
\end{aligned}
$$

Thus, $f\left(\mu^{g}\right)\left(x^{\prime}+y^{\prime}\right) \geq \min \left\{f\left(\mu^{g}\right)\left(x^{\prime}\right), f\left(\mu^{g}\right)\left(y^{\prime}\right)\right\}$.

Also, $f\left(\mu^{g}\right)\left(r x^{\prime}\right)=f\left(\mu^{g}\right)(r f(x))=f\left(\mu^{g}\right)(f(r x))$

$$
\begin{aligned}
& =\mu^{g}(r x)[\because f \text { is a bijective G-module homomorphism }] \\
& \geq \mu^{g}(x)\left[\because \mu^{g} \text { is a FSM of M }\right] \\
& =f\left(\mu^{g}\right)(f(x))=f\left(\mu^{g}\right)\left(x^{\prime}\right)
\end{aligned}
$$

Thus $f\left(\mu^{g}\right)\left(r x^{\prime}\right)=f\left(\mu^{g}\right)\left(x^{\prime}\right)$.

Therefore, $f\left(\mu^{g}\right)$ and so $(f(\mu))^{g}$ is a fuzzy submodule of $\mathbf{M}^{\prime}$.

Hence $f(\mu)$ is a fuzzy G-submodule of $\mathbf{M}^{\prime}$.

Theorem (4.5) Let $\mathrm{M}$ and $\mathrm{M}^{\prime}$ be Z-modules on which $\mathrm{G}$ acts and let $f$ be a G-module homomorphism from $\mathrm{M}$ into $\mathrm{M}^{\prime}$. If $v$ be a G-invariant fuzzy submodule of $\mathrm{M}^{\prime}$, then $f^{-1}(v)$ is a G-invariant fuzzy submodule of $\mathrm{M}$.

Proof. Since $v$ is G-invariant fuzzy submodule of $\mathrm{M}^{\prime}$. Therefore, $v^{g}=v$, for all $g \in \mathrm{G}$.

Now, $\left(f^{-1}(v)\right)^{g}=f^{-1}\left(v^{g}\right)=f^{-1}(v), \forall g \in \mathrm{G}$.

Therefore, $f^{-1}(v)$ is G-invariant fuzzy submodule of $\mathrm{M}$.

Theorem (4.6) Let $\mathrm{M}$ and $\mathrm{M}^{\prime}$ be Z-modules on which $\mathrm{G}$ acts and let $f$ be a bijective G-module homomorphism from $\mathrm{M}$ into $\mathrm{M}^{\prime}$. If $\mu$ is a $\mathrm{G}$-invariant fuzzy submodule of $\mathrm{M}$, then $f(\mu)$ is a G-invariant fuzzy submodule of $\mathrm{M}^{\prime}$.

Proof. Since $\mu$ is G-invariant fuzzy submodule of $\mathrm{M}^{\prime}$. Therefore, $\mu^{g}=\mu$, for all $g \in \mathrm{G}$.

Now, $(f(\mu))^{g}=f\left(\mu^{g}\right)=f(\mu), \forall g \in \mathrm{G}$.

Hence $f(\mu)$ is G-invariant fuzzy submodule of $\mathbf{M}^{\prime}$.

\section{Conclusion}

In this paper, the notion of fuzzy G-submodule of a Z-module is defined and discussed. It has been proved that 
every fuzzy G-module is a fuzzy module but the converse is not true in general. It has also been proved that intersection and Cartesian product of two fuzzy G-submodules are fuzzy G-submodules. The notions of fuzzy prime submodule, fuzzy G-prime submodule, fuzzy semi prime submodule and fuzzy G-semi prime submodule are introduced and discussed. We have observed that intersection of two fuzzy prime submodules needs not be a fuzzy prime submodule; however intersection of two fuzzy prime submodules is always a fuzzy semi prime submodule. The notions of G-invariant fuzzy subset (submodule) and G-invariant fuzzy prime (G-prime) submodule of Z-module are also introduced and discussed. We have proved that sum and product of two G-invariant fuzzy sub-modules are G-invariant fuzzy submodules.

\section{References}

[1] Zadeh, L.A. (1965) Fuzzy Sets. Information and Control, 8, 338-353. http://dx.doi.org/10.1016/S0019-9958(65)90241-X

[2] Negoita, C.V. and Ralescu, D.A. (1975) Applications of Fuzzy Sets and System Analysis, Birkhous Basel. http://dx.doi.org/10.1007/978-3-0348-5921-9

[3] Han, J.-C. (2005) Group Actions in a Regular Ring. Bulletin of the Korean Mathematical Society, 42, 807-815.

[4] Sharma, R.P. and Sharma, S. (1998) Group Action on Fuzzy Ideals. Communications in Algebra, 26, 4207-4220. http://dx.doi.org/10.1080/00927879808826406

[5] Sharma, R.P., Gupta, J.R. and Arvind (2000) Characterization of G-Prime Fuzzy Ideals in a Ring-An Alternative Approach. Communications in Algebra, 28, 4981-4987. http://dx.doi.org/10.1080/00927870008827135

[6] Rosenfeld, A. (1971) Fuzzy Groups. Journal of Mathematical Analysis and Application, 35, 512-517. http://dx.doi.org/10.1016/0022-247X(71)90199-5

[7] Atanassov, K.T. (1986) Intuitionistic Fuzzy Sets. Fuzzy Sets and Systems, 20, 87-96. http://dx.doi.org/10.1016/S0165-0114(86)80034-3

[8] Xu, C.Y. (2008) Intuitionistic Fuzzy Modules and Their Structures. Lecture Notes in Computer Science, 5227, $459-467$. http://dx.doi.org/10.1007/978-3-540-85984-0_55

[9] Saikia, H.K. and Kalita, M.C. (2009) On Annihilator of Fuzzy Subsets of Modules. International Journal of Algebra, 3 , 483-488.

[10] Majumdar, S. (1990) Theory of Fuzzy Modules. Bulletin of Calcutta Mathematical Society, 82, 395-399.

[11] Mukherjee, T.K., Sen, M.K. and Roy, D. (1996) On Fuzzy Submodules and Their Radicals. Journal of Fuzzy Mathematics, 4, 549-557.

[12] Zheng, P.F. (1988) Fuzzy Quotient Modules. Fuzzy Sets and Systems, 28, 85-90. http://dx.doi.org/10.1016/0165-0114(88)90118-2

[13] Isaac, P., and John, P.P. (2011) On Intuitionistic fuzzy Submodules of a Module. International journal of Mathematical Sciences and Applications, 1, 1447-1454.

[14] Rahman, S. and Saikia, H.K. (2012) Some Aspects of Atannassoves Intuitionistic Fuzzy Submodules. International Journal of Pure and Applied Mathematics, 77, 369-383.

[15] Rajkhowa, K.K. and Saikia, H.K. (2015) Center of Intersection Graph of Fuzzy Submodules of Modules. Fuzzy Information and Engineering, 7, 49-59. http://dx.doi.org/10.1016/j.fiae.2015.03.004

[16] Sharma, P.K. (2013) ( $\alpha$, $\beta$ )-Cut of Intuitionistic Fuzzy Modules-II. International Journal of Mathematical Sciences and Applications, 3, 11-17.

[17] Sharma, P.K., and Bahl, A. (2012) Translates of Intuitionistic Fuzzy Submodules. International Journal of Mathematicals Sciences, 11, 277-287.

[18] Mordeson, J.N. and Malik, D.S. (1998) Fuzzy Commutative Algebra. World Scientific, Singapore.

[19] Zheng, P.F. (1987) Fuzzy Finitely Generated Modules. Fuzzy Sets and Systems, 21, 105-113. http://dx.doi.org/10.1016/0165-0114(87)90156-4

[20] Kumar, R., Bhambri S.K. and Kumar P. (1995) Fuzzy Submodules: Some Analogues and Deviations. Fuzzy Sets and Systems, 70, 125-130. http://dx.doi.org/10.1016/0165-0114(94)00260-E 